Case Report

\title{
Importance of Strain Echocardiography and Cardiac Magnetic Resonance for the Early Diagnosis of Myocarditis
}

\author{
Victor Rodrigues Ribeiro Ferreira ${ }^{1,2}$, Maria Christiane Valéria Braga Braile-Sternieri', \\ Eliana Migliorini Mustafa ${ }^{1}$, Sofia Braile Sabino ${ }^{1}$, Cibele Olegário Vianna Queiroz, \\ Bethina Canaroli Sbardellini ${ }^{1}$, Giovanni Braile Sternieri ${ }^{1}$, Luiza Braile Verdi ${ }^{1}$, \\ Idiberto José Zotarelli Filho ${ }^{1, *}$, Domingo Marcolino Braile ${ }^{1}$ \\ ${ }^{1}$ Domingo Braile Institute, Institute of Cardiology, Sao Jose do Rio Preto, Brazil \\ ${ }^{2}$ Faceres, Medical School of São Jose do Rio Preto, Sao Jose do Rio Preto, Brazil
}

Email address:

scientific@institutodomingobraile.com.br (I. J. Z. Filho)

${ }^{*}$ Corresponding author

\section{To cite this article:}

Victor Rodrigues Ribeiro Ferreira, Maria Christiane Valéria Braga Braile-Sternieri, Eliana Migliorini Mustafa, Sofia Braile Sabino, Cibele Olegário Vianna Queiroz, Bethina Canaroli Sbardellini, Giovanni Braile Sternieri, Luiza Braile Verdi, Idiberto José Zotarelli Filho, Domingo Marcolino Braile. Importance of Strain Echocardiography and Cardiac Magnetic Resonance for the Early Diagnosis of Myocarditis. Pathology and Laboratory Medicine. Vol. 3, No. 2, 2019, pp. 23-27. doi: 10.11648/j.plm.20190302.11

Received: September 5, 2019; Accepted: October 4, 2019; Published: December 2, 2019

\begin{abstract}
Introduction: Myocarditis (MC) can result from a variety of infectious and non-infectious causes, and the secondary $\mathrm{MC}$ due to viral infection is the most prevalent form. Acute and nonfatal MC, on the other hand, has a more indolent presentation with mortality up to $20.0 \%$ in the first year and $55.0 \%$ at 11 years based on the initial diagnosis of acute MC. The diagnostic evaluation of MC is done initially through clinical suspicion, together with non-invasive diagnostic methods. In this sense, we highlight the Echocardiography with Strain. Objective: to present the importance of the early diagnosis of myocarditis through the images obtained by the Strain Echocardiography and Cardiac Magnetic Resonance. Case report: The CBB patient, 25 years old, female, with no known diagnosis, reports that two weeks ago the patient had started upper airway infection with partial improvement after specific antimicrobial treatment. The hemogram revealed normocytic and normochromic anemia and leukocytosis with predominance of segmented. C-reactive protein was elevated and markers of myocardial ischemia were also altered. Strain echocardiography revealed segmental alterations of the endomyocardial deformation with reduction of the longitudinal Strain in the middle inferolateral and middle anterolateral segments. Cardiac magnetic resonance revealed late focal mesoepicardial enhancement, of small extension, in the midbasal inferolateral segment, suggestive of MC. Final considerations: In conclusion the study made it possible to know that the early diagnosis of myocarditis through the image obtained by the Strain Echocardiography showed the inflammatory process in the same region observed by cardiac magnetic resonance imaging. This was added to the hemogram, reactive protein, and to the markers of myocardial ischemia.
\end{abstract}

Keywords: Myocarditis, Cardiac Magnetic Resonance, Strain Echocardiography, Diagnosis

\section{Introduction}

Myocarditis (MC) is defined as an inflammatory myocardial disease, secondary to infectious, autoimmune or toxic agents, characterized by a histopathological process revealing inflammation of the myocardium with necrosis and degeneration of myocytes in the absence of myocardial ischemia $[1,2]$.

MC may be due to several infectious and non-infectious causes, and the secondary MC due to viral infection is the most prevalent form [2]. The viral MC can be divided into the acute, subacute and chronic phase. The knowledge of this temporal and pathophysiological evolution is important in the diagnostic definition and in the therapeutic possibilities [2,3].

Acute and nonfatal MC, on the other hand, has a more lazy 
presentation with mortality up to $20.0 \%$ in the first year and $55.0 \%$ at 11 years based on the initial diagnosis of acute MC [2, $3]$. The scope of the disease includes significant morbidity of dilated cardiomyopathy and mortality from sudden cardiac death. Up to $12.0 \%$ of sudden cardiac deaths are attributed to $\mathrm{MC}[2]$.

The true incidence of MC is difficult to estimate due to factors such as diverse clinical presentation, poorly performing an endomyocardial biopsy, and lack of sensitive and standardized histologic criteria. It manifests a higher prevalence in males, mainly young adults, being one of the main causes of sudden death in people under 40 years of age and in children [4-6]. In children who survived MC, evolution was observed after 12 years, with higher mortality and need for heart transplantation [7].

Causation can be attributed to an internal trigger such as an autoimmune reaction or external factors, including toxins, drugs or infections $[8,9]$. The fulminant MC is marked by an identifiable onset within 2 weeks of hemodynamic compromise and has a high survival rate $(90.0 \%)$ if diagnosed and treated in a timely manner [10].

The diagnostic evaluation of $\mathrm{MC}$ is done initially through clinical suspicion, together with non-invasive diagnostic methods. In this sense, Strain Echocardiography (SE) is a technique that evaluates the measurement of the deformation that undergoes the myocardial wall, during the cardiac cycle, in the longitudinal direction and circumferential directions, by the apical projection and transverse axis of the left ventricle, being the deformation rate quantification of this deformation [8-10]. The deformation of the myocardial wall is analyzed by the movement of points in the two-dimensional image of the myocardium, determining the velocity with which they approach or move away, throughout the cardiac cycle [10].

In addition, a number of recent studies have shown the importance of Cardiac Magnetic resonance (CMR) in the acute or chronic phase of MC by techniques of magnetic signal weighting and late enhancement [11-14]. The multifocal enhancement pattern, together with the signal increase in the acute phase of the disease, allows a sensitivity of $76.0 \%$, specificity of $96.0 \%$ and accuracy of $85.0 \%$ in the diagnosis of the disease, being superior to the myocardial scintigraphy with gallium and allowing to consider the method as one of the first-line choices in the investigation of this disease [14].

In this sense, CMR allows us to identify both the inflammatory myocardial injury of the acute and subacute phases and the cicatricial lesions present in the chronic phase of the disease [15]. The three major CMR techniques used in the characterization of myocardial injury in $\mathrm{MC}$ patients are T2-weighted sequences, early global myocardial enhancement and the late enhancement technique [16]. The images acquired by the T2-weighted sequences allow the assessment of myocardial edema secondary to the inflammatory process in patients with acute $\mathrm{MC}$ and can be both regional and global $[16,17]$.

Therefore, the present work sought to present the importance of the early diagnosis of myocarditis through the images obtained by the Strain Echocardiography and Cardiac Magnetic Resonance.

\section{Case Report}

The CBB patient, 25 years old, female, with no known illness, reports that two weeks ago the patient had started upper airway infection with partial improvement after specific antimicrobial treatment.

He complained of chest discomfort and dyspnea on exertion with few hours of evolution, when he sought medical care in the emergency room, where he was submitted to complementary analysis by biochemical markers. The hemogram revealed normocytic and normochromic anemia and leukocytosis with a predominance of segmented. C-reactive protein was elevated and markers of myocardial ischemia were also altered: CKMB $16.2 \mathrm{ng} / \mathrm{mL}(\mathrm{NL}<5)$ and highly sensitive troponin T $217.30 \mathrm{pg} / \mathrm{mL}(\mathrm{NL}<14)$.

The patient was then submitted to Strain Echocardiography, which revealed segmental alterations of the endomyocardial deformation with reduction of the longitudinal Strain in the middle and lowers anterolateral segments (Figures 1 and 2).

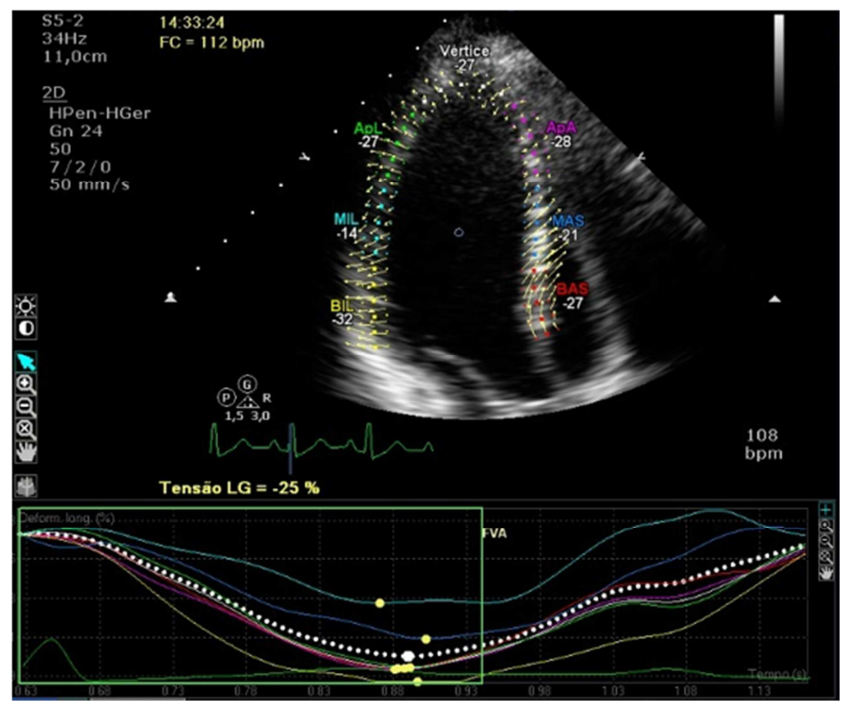

Figure 1. Strain Echocardiography results showing segmental changes of the endomyocardial deformation with reduction of the longitudinal Strain in the middle inferolateral and middle anterolateral segments.

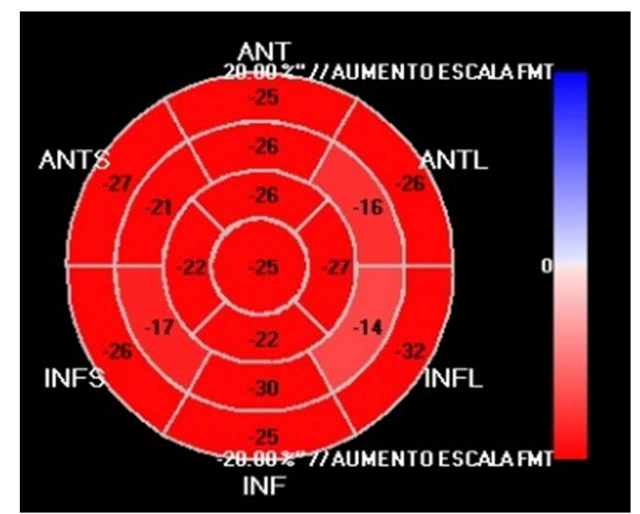

Figure 2. Strain Echocardiography results showing peak systolic blood pressure. Heart rate $=114 \mathrm{bpm}$. 
However, the overall longitudinal Strain was within the limits of normality. With the diagnostic hypothesis of focal $\mathrm{MC}$, the patient was followed up for hospital admission, when Cardiac Magnetic Resonance was performed, which revealed focal mesoepicardial late enhancement of the mid-base inferolateral segment of non-ischemic pattern suggestive of MC (Figure 3).

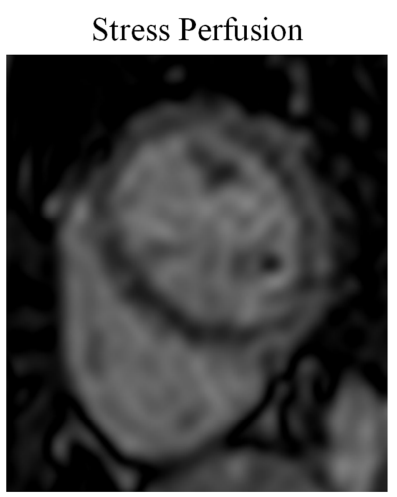

Post-Stress Perfusion

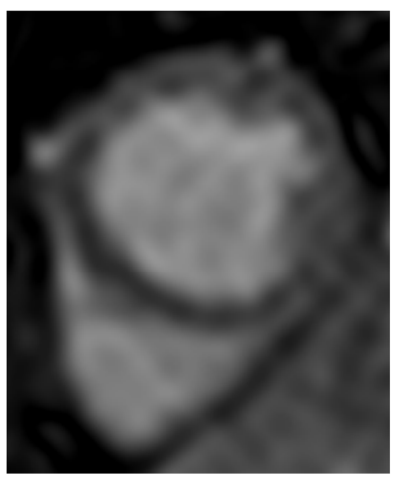

Stress Perfusion

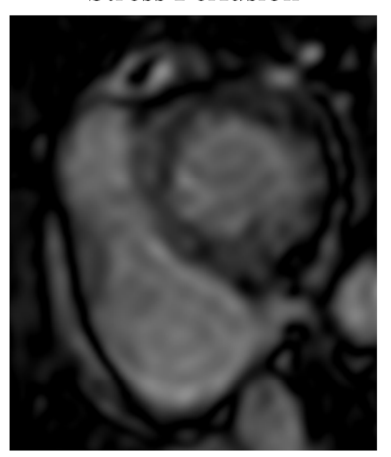

Post-Stress Perfusion

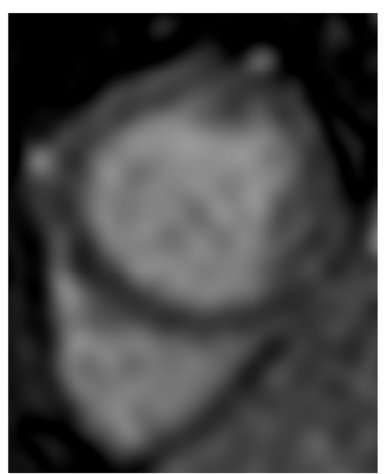

Figure 3. Results of cardiac magnetic resonance showing focal mesoepicardial late enhancement, of small extension, in the midbasal inferolateral segment.

The patient was hospitalized for three days, remission of symptoms and improvement of serum markers of myocardial injury: CKMB mass $1.2 \mathrm{ng} / \mathrm{mL}$. A new Strain Echocardiography was performed after one week of treatment that revealed normalization of the previous alterations of endomyocardial deformation.

The description of Strain's images revealed a mean overall longitudinal Strain within the limits of normalities with segmental, medial inferolateral and anterolateral mean reduction.

\section{Result and Discussion}

The present case report evidenced the early diagnosis of myocarditis (MC) through the images obtained by the echo-strain that the alterations of the inflammatory process occurred in the same region observed by cardiac magnetic resonance (CMR). In addition, the hemogram showed normocytic and normochromic anemia and leukocytosis with a predominance of segmented. C-reactive protein was elevated and markers of myocardial ischemia were also altered.

In the evaluation of MC patients, CMR allows the identification of both acute and subacute inflammatory myocardial injury, as well as the cicatricial lesions, frequently present in the chronic phase of the disease [1-3]. The three main CMR techniques used in the characterization of myocardial injury in MC patients are T2-weighted sequences (T2 imaging), early global myocardial enhancement and the late enhancement technique [3]. The images acquired by the T2-weighted sequences allow the evaluation of myocardial edema secondary to the inflammatory process in patients with acute $\mathrm{MC}$ and can be both regional and global [3].

Thus, CMR is currently the main complementary noninvasive diagnostic method and allows visualization of the location, activity, and extent of inflammation and fibrosis in the myocardium [3, 4]. The late enhancement arrangement found in the myocardium is usually epicardial and/or mesocardial, preserving the endocardium, a typical pattern that excludes ischemic cause, presenting a transmural or subendocardial arrangement respecting the coronary vascularization [4].

In this context, the late enhancement has a prognostic value when used in the follow-up, since patients who show a reduction of the enhancement at 3 months present better long-term survival $[5,6]$. CMR can also be used to support endomyocardial biopsy guidance, increasing the positivity rate when performed on the wall with present enhancement from 9.0 to $90.0 \%$ [7].

The Strain Echocardiography has become the most important means to detect decreased ventricular function in suspected MC, even when subclinical [18]. Allows the measurement of systolic and diastolic ventricular function, myocardial segmental function, detection of viability in hibernating walls, differentiation of athlete's hypertrophic cardiomyopathy, and measurement of contraction timing changes [8]. In addition, because it represents an examination with minimal risks to patients, it becomes an attractive non-invasive alternative [8].

The deformation of the myocardial fibers is a marker of the onset of cardiac function disorders caused by diseases such as hypertension, diabetes, and ischemia [9, 10, 17].

Also, acute myocarditis should be considered in patients with a recent onset of heart failure or arrhythmia. Fulminant myocarditis is a distinct entity characterized by the sudden onset of severe congestive heart failure or cardiogenic shock, usually following an influenza-like disease, parvovirus B19, human herpesvirus 6 , coxsackievirus and adenovirus being the most responsible viruses. Thus, early recognition of patients who progress rapidly to refractory heart failure is required. Immunosuppression of self-reactive or immunostimulant myocarditis, such as interferons for chronic viral myocarditis, may be interesting, but its potential therapeutic role requires further investigation [hekimian].

In addition, viral myocarditis remains a prominent infectious-inflammatory disease in lifelong patients. The condition presents a number of challenges, including varying modes of clinical presentation, a variety of time points at 
which patients draw attention, a variety of diagnostic approaches, a spectrum of clinical courses, and unstable perspectives on therapy in different patient contexts. and in the face of different viral pathogens [fung].

\section{Conclusion}

In conclusion, the work made it possible to know that the early diagnosis of myocarditis through the image obtained by the Strain Echocardiography showed the inflammatory process in the same region observed by cardiac magnetic resonance imaging. This was added to the hemogram, reactive protein, and to the markers of myocardial ischemia. Future directions include large prospective studies.

\section{Recommendations}

We recommend the use of The Strain Echocardiography has become the most important means to detect decreased ventricular function in suspected MC. In addition, because it represents an examination with minimal risks to patients, it becomes an attractive non-invasive alternative.

\section{Acknowledgements}

The work was supported by Domingo Braile Institute - São José do Rio Preto - SP, Brazil.

\section{Declaration of Potential Conflict of Interest}

The authors declare no conflict of interest.

\section{References}

[1] Eyer-Silva WA, Rosa da Silva GA, da Cunha Pinto JF. Acute myocarditis after switch to dolutegravir: a reminder of potential toxicity of integrase inhibitor-including HAART. AIDS. 2019 Nov 1; 33 (13): 2105-2107. doi: 10.1097/QAD.0000000000002322.

[2] Pedrotti P, Pedretti S, Imazio M, Quattrocchi G, Sormani P, Milazzo A, Quarta G. Clinical applications of cardiac magnetic resonance imaging: coronary heart disease, myocarditis, pericardial diseases, arrhythmias, valvular heart disease, congenital heart disease and cardiac masses. G Ital Cardiol (Rome). 2019 Jan; 20 (1): 8-19. doi: 10.1714/3079.30716.

[3] Gannon MP, Schaub E, Grines CL, Saba SG. State of the art: Evaluation and prognostication of myocarditis using cardiac MRI. J Magn Reson Imaging. 2019 Jan 13. doi: 10.1002/jmri.26611.

[4] Tornvall P, Brolin EB, Caidahl K, et al. The value of a new cardiac magnetic resonance imaging protocol in Myocardial Infarction with Nonobstructive Coronary Arteries (MINOCA) - A case-control study using historical controls from a previous study with similar inclusion criteria. BMC Cardiovasc Disord 2017; 17: 199.
[5] Chopra H, Arangalage D, Bouleti C, et al. Prognostic value of the infarctand non-infarct like patterns and cardiovascular magnetic resonance parameters on long-term outcome of patients after acute myocarditis. Int J Cardiol 2016; 212: 63-69.

[6] De Lazzari M, Zorzi A, Baritussio A, et al. Relationship between T-wave inversion and transmural myocardial edema as evidenced by cardiac magnetic resonance in patients with clinically suspected acute myocarditis: Clinical and prognostic implications. J Electrocardiol 2016; 49: 587-595.

[7] Hinojar R, Nagel E, Puntmann VO. T1 mapping in myocarditis-Headway to a new era for cardiovascular magnetic resonance. Expert Rev Cardiovasc Ther 2015; 13: 871-874.

[8] Lurz P, Luecke C, Eitel I, et al. Comprehensive cardiac magnetic resonance imaging in patients with suspected myocarditis: The MyoRacerTrial. J Am Coll Cardiol 2016; 67: 1800-1811.

[9] Del Castlho JM and Herszkowicz N. Strain bidimensional (X-Strain): utlização do método para avaliação de cardiopatas. Rev Bras Ecocardiogr. 2008; 21: 29-25.

[10] Delgado V, Mollema SA, Ypenburg C, Tops LF, van der Wall EE, Schalij MJ and Bax JJ. Relaton between global lef ventricular longitudinal strain assessed with novel automated functon imaging and biplane lef ventricular ejecton fracton in patents with coronary artery disease. J Am Soc Echocardiogr. 2008; 21: 1244-50.

[11] Lo Q, Hee L, Batumalai V, Allman C, MacDonald P, Lonergan D, Delaney GP and Thomas L. Strain Imaging Detects Dose-Dependent Segmental Cardiac Dysfuncton in the Acute Phase Afer Breast Irradiaton. Int J Radiat Oncol Biol Phys. 2017; 99: 182-190.

[12] Mangion K, McComb C, Auger DA, Epstein FH and Berry C. Magnetc Resonance Imaging of Myocardial Strain Afer Acute ST-SegmentElevaton Myocardial Infarcton: A Systematc Review. Circ Cardiovasc Imaging. 2017; 10.

[13] Mann DL, Zipes DP, Libby P, Bonow RO, Braunwald E. Braunwald's heart disease: A textbook of cardiovascular medicine, 10th ed. Philadelphia: Elsevier/Saunders; 2015.

[14] Luetkens JA, Homsi R, Dabir D, et al. Comprehensive cardiac magnetic resonance for short-term follow-up in acute myocarditis. J Am Heart Assoc 2016; 5.

[15] Gupta S, Markham DW, Drazner MH, Mammen PP. Fulminant myocarditis. Nat Clin Pract Cardiovasc Med 2008; 5: 693-706.

[16] Angelini A, Calzolari V, Calabrese F, et al. Myocarditis mimicking acute myocardial infarction: Role of endomyocardial biopsy in the differential diagnosis. Heart 2000; 84: 245-250.

[17] Babu-Narayan SV, McCarthy KP, Ho SY, Magee AG, Kilner PJ, Sheppard MN. Images in cardiovascular medicine. Myocarditis and sudden cardiac death in the young: Extensive fibrosis suggested by cardiovascular magnetic resonance in vivo and confirmed post mortem. Circulation 2007; 116: e122-125.

[18] Cooper LT Jr, Keren A, Sliwa K, Matsumori A, Mensah GA. The global burden of myocarditis. Global Heart 2014; 9: 121129.

[19] Haaf P, Buser PT. Map to the future of cardiac magnetic resonance in myocarditis. Eur Heart J Cardiovasc Imaging. 2017 Jul 1; 18 (7): 752-753. doi: 10.1093/ehjci/jex024. 
[20] Hékimian G, Combes A. Myocarditis]. Rev Med Interne. 2017 Aug; 38 (8): 531-538. doi: 10.1016/j.revmed.2016.12.022. Epub 2017 Feb 2.
[21] Fung G, Luo H, Qiu Y, Yang D, McManus B. Myocarditis. Circ Res. 2016 Feb 5; 118 (3): 496-514. doi: 10.1161/CIRCRESAHA.115.306573. 